\title{
COVID-19 in Turkish health care workers practicing chest medicine
}

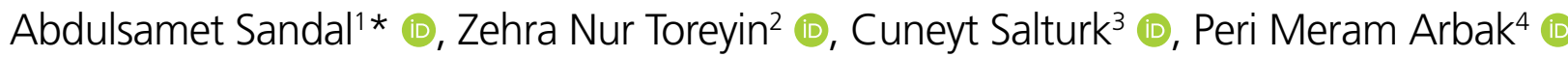

\section{SUMMARY}

OBJECTIVE: This study aimed to evaluate the data of Turkish health care workers practicing chest medicine on their coronavirus disease 2019 (COVID-19) status and related parameters.

METHODS: This descriptive study included online survey data that the Turkish Thoracic Society conducted with its members in two phases starting in June and December 2020. The 33-item survey included demographic data, smoking status, the presence of any chronic diseases, occupation, working status, and non-work-related and work-related COVID-19 exposure characteristics.

RESULTS: Of 742 responses, 299 (40.3\%) reported that they had contracted COVID-19. The second survey detected a higher frequency of health care workers who had contracted COVID-19 (12.1\% versus $57.4 \%, \mathrm{p}<0.001)$ than the first survey. The analysis of the association between study parameters and COVID-19 in health care workers using logistic regression revealed statistical significance with working at the onset of the outbreak (OR 3.76, 95\% Cl 1.09-12.98, $\mathrm{p}=0.036)$, not working at the time of survey $(\mathrm{OR} 5.69,95 \% \mathrm{Cl} 3.35-9.67$, $\mathrm{p}<0.001)$, COVID-19 history in colleagues (OR 2.27, 95\% CI 1.51-3.41, $\mathrm{p}<0.001)$, any non-work-related COVID-19 exposure (OR 4.72 , $95 \% \mathrm{Cl} 2.74-8.14, \mathrm{p}<0.001)$, COVID-19 exposure at home (OR 6.52, 95\% Cl 3.52-12.08, p<0.001), and COVID-19 history in family members (OR 8.16, 95\% CI 5.52-12.08, p<0.001) after adjusting for age and sex. The study also observed an inverse relationship between the use of aprons and goggles and COVID-19 in health care workers.

CONCLUSION: Occupational and nonoccupational characteristics are related to COVID-19 in health care workers practicing chest medicine. Therefore, active surveillance to detect health care workers contracting COVID-19 and to document and control occupational and nonoccupational risks should be provided.

KEYWORDS: COVID-19. Occupational health. Health personnel. Occupational medicine. Occupational diseases.

\section{INTRODUCTION}

Coronavirus disease 2019 (COVID-19) emerged toward the end of 2019 and was declared a pandemic by the World Health Organization (WHO) on March 11, 2020, due to its rapid global spread $^{1}$. However, the risk of infection has not been the same for all people. Indeed, workers with essential jobs, also called frontline workers, faced a higher risk than the general population during the pandemic ${ }^{2}$. Of those, health care workers (HCWs) have encountered occupational risks related to COVID-193.
In Turkey, the Minister of Health officially announced the first COVID-19 diagnosis on March 11, 2020 ${ }^{4}$. Since then, the demand for health care services has progressively increased while the number of cases has grown ${ }^{5}$, similar to the situation in other countries. At the initial phase of the outbreak, the Ministry of Health of Turkey defined a pandemic referral hospital as a hospital with a tertiary intensive care unit and employing specialists with at least any two specialties of internal medicine, infectious diseases, and chest medicine ${ }^{4,6}$. Thus, being among

\footnotetext{
${ }^{1}$ Ankara Occupational and Environmental Diseases Hospital, Occupational Diseases Clinic - Ankara, Turkey.

${ }^{2}$ Adana City Training and Research Hospital, Occupational Diseases Clinic - Adana, Turkey.

${ }^{3}$ Yeni Yuzyil University, Department of Chest Diseases - Istanbul, Turkey.

${ }^{4}$ Duzce University, Department of Chest Diseases - Duzce, Turkey.

*Corresponding author: asandal@hotmail.com.tr

Conflicts of interest: the authors declare there are no conflicts of interest. Funding: none.

Received on July 13, 2021. Accepted on August 11, 2021.
} 
the essential members of the health care services during the outbreak in Turkey meant HCWs practicing chest medicine have faced occupational risks and contracted COVID-19 since the early days of the pandemic. The Turkish Thoracic Society (TTS), as one of the principal societies for Turkish HCWs working in chest medicine, has asked its members about their COVID-19 status and related occupational and nonoccupational characteristics via online surveys. This study aimed to evaluate COVID-19 status and related parameters of Turkish HCWs practicing chest medicine through the data collected by the TTS.

\section{METHODS}

\section{Study design, study population, and data collection}

This descriptive study included the data obtained by the online surveys which the TTS conducted with its members in two consecutive phases to monitor their COVID-19 status and related parameters. This study was performed in accordance with the principles of the Declaration of Helsinki. The study protocol was approved by the Duzce University Ethics Board for Noninterventional Health Research (Decision No. 2021/37).

The 33-item survey prepared by the Occupational Lung Diseases Working Group of TTS included demographic information, smoking status, the presence of any chronic diseases, occupation, current working status, working status at the onset of the outbreak, and the characteristics of work-related and non-work-related COVID-19 exposure. The characteristics of non-work-related COVID-19 exposure included the place (home or other) of exposure and if any household member had contracted COVID-19. The characteristics of work-related COVID-19 exposure included the status of work-related COVID-19 exposure according to hospital division (outpatient clinics, wards, COVID-19 triage area, intensive care unit, emergency department, and other departments), any exposure to secretions from infected patients, COVID-19 history in colleagues, and the use of personal protective equipment (PPE), namely, disposable gloves, surgical masks, respirators, facial protectors, goggles, aprons, and gowns. The participants were asked if they contracted COVID-19. The HCWs contracted COVID-19 were also questioned about the symptom status, types of symptoms, the date, and method (i.e., polymerase chain reaction $[\mathrm{PCR}]$, serology, and clinical and/or radiological) of COVID-19 diagnosis. In addition, the second survey asked if the respondent had participated in the initial survey.

The web links to the online surveys were sent via email by the TTS on June 1,2020, during the first phase, and on
December 8, 2020, during the second phase. The TTS members receiving the email totaled 6,103 and 6,325 in June 2020 and December 2020, respectively. The first survey remained open for 5 weeks, with four reminder emails sent weekly. The second survey remained open until the end of January 2021, and seven reminders were sent to the members. The analysis excluded duplicate records resulting from re-sent answers and second survey responses reporting prior participation in the first survey. The number of new diagnoses of COVID-19 cases per week in Turkey was derived from the WHO COVID-19 Dashboard ${ }^{7}$.

\section{Statistical analysis}

The descriptive statistics were presented as mean \pm standard deviation or median and minimum-maximum for continuous variables and as numbers and percentages for categorical variables. The chi-square test compared categorical variables. Crude and age- and sex-adjusted logistic regression analyses evaluated the relationship between parameters and COVID-19 status, and the odds ratios (ORs) with a $95 \%$ confidence interval $(95 \% \mathrm{CI})$ values were calculated. The type I error was accepted as 0.05 for all analyses. All statistical analyses were performed using IBM SPSS for Windows version 22.0 (IBM Corp., Armonk, NY, USA).

\section{RESULTS}

Of 868 responses, 4 duplicates and 122 responses to the second survey reporting prior participation to the first survey were excluded. In total, 742 responses (280 in the first survey and 462 in the second survey) were included, and 475 (64.0\%) were females. The median age of 716 participants reported their age was 43 (min-max, 22-73). The number of participants who reported their home province was 703 (94.7\%). Of those respondents, 209 (29.7\%) were from Istanbul, 108 (15.4\%) from Ankara, and 70 (10.0\%) from Izmir. The total number of participants reporting that they had contracted COVID-19 was 299 (40.3\%). Compared to the first survey, a higher frequency of HCWs contracted COVID-19 was detected in the second survey $(12.1 \%$ versus $57.4 \%, \mathrm{p}<0.001)$.

Figure 1 shows the weekly distribution of 278 (93.0\%) HCWs who contracted COVID-19 and reported the date of diagnosis, together with the weekly number of new diagnoses of COVID-19 cases in Turkey. The highest number of diagnoses stood at 30 in the week of November 23, 2020.

In the comparison of demographic and clinical characteristics between HCWs who had contracted COVID-19 and HCWs without a history of COVID-19 (Table 1), the characteristics with significantly higher frequency in HCWs having contracted COVID-19 were having comorbid asthma, 


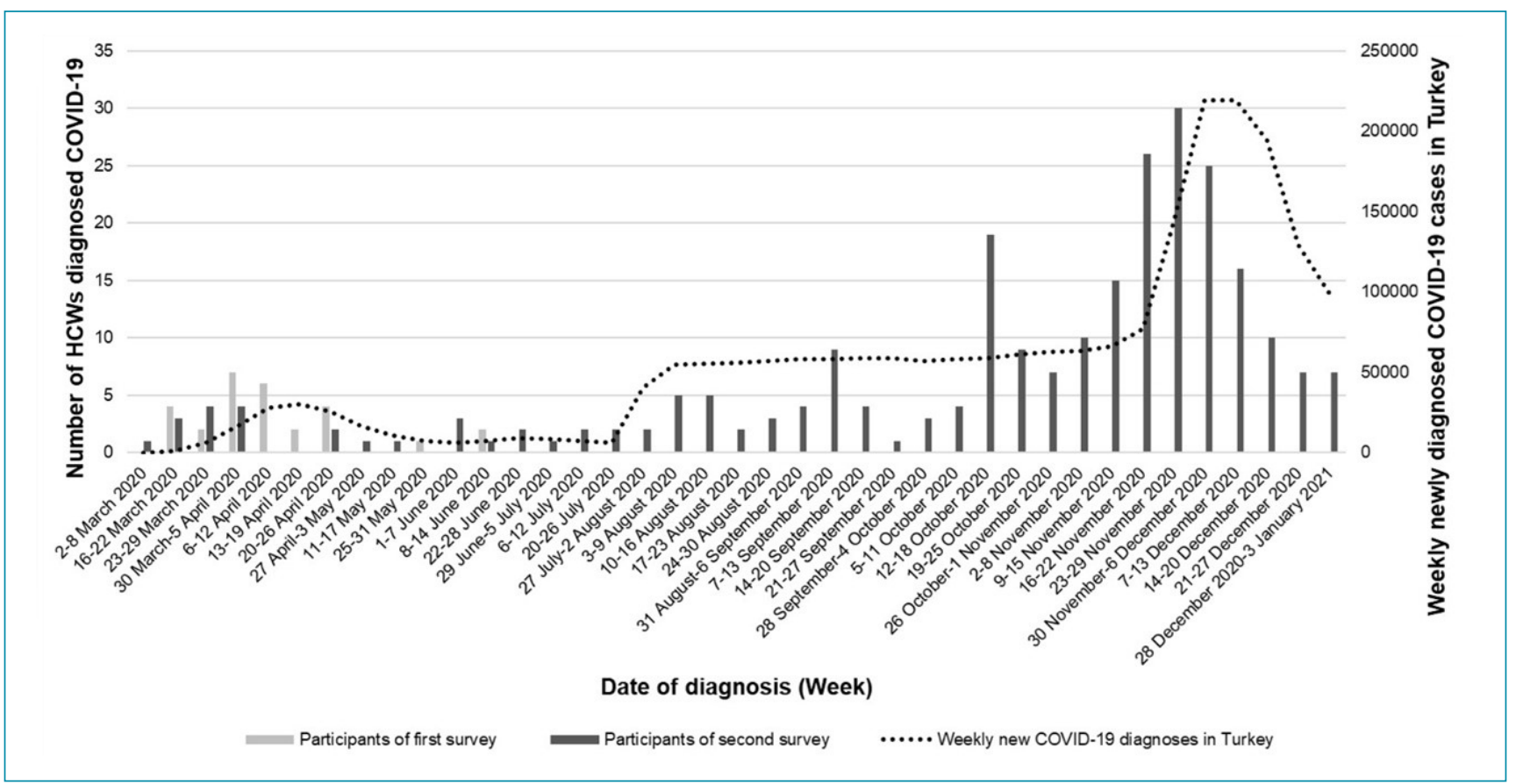

Figure 1. COVID-19: coronavirus disease 2019; HCW: healthcare worker. The distribution of HCWs with COVID-19 according to the week of diagnosis. Twenty-one participants who did not report the date of COVID-19 diagnosis were not included.

non-work-related COVID-19 exposure, COVID-19 exposure at home, COVID-19 history in household members, working at the onset of the outbreak, not working at the time of survey, and COVID-19 history in colleagues. There was also a statistically significant difference according to the smoking status. In terms of PPE, the use of aprons and goggles proved lower in HCWs with COVID-19. Similar comparisons were performed for each survey phase, and the results are presented in Table 1.

In terms of symptoms, 177 (59.2\%) HCWs with COVID-19 reported that their infection was asymptomatic. The frequencies of symptoms were 70 (23.4\%) for a cough, 66 (22.1\%) for headache, 59 (19.7\%) for loss of smell and/or taste, 47 (15.7\%) for fever, $42(14.0 \%)$ for nasal congestion and/or rhinorrhea, $40(13.4 \%)$ for shortness of breath, $29(9.7 \%)$ for chest pain, $21(7.0 \%)$ for nausea/vomiting, 19 (6.4\%) for diarrhea, and $16(5.4 \%)$ for muscle or joint pain. The diagnostic method was PCR in 215 (71.9\%) and positive serology in 40 (13.4\%), but 44 (14.7\%) were diagnosed clinically and/or radiologically.

The association between selected parameters and COVID-19 in HCWs underwent evaluation with crude and age- and sex-adjusted logistic regression analysis, and working at the onset of the outbreak (OR 3.76, 95\%CI 1.09-12.98), not working at the time of survey (OR 5.69, 95\%CI 3.35-9.67), COVID-19 history in colleagues (OR 2.27, 95\%CI 1.51-3.41), any non-work-related COVID-19 exposure (OR 4.72, 95\%CI 2.74-8.14), COVID-19 exposure at home (OR 6.52, 95\%CI
3.52-12.08), and COVID-19 history in family members (OR $8.16,95 \%$ CI $5.52-12.08$ ) bore a significant relationship to COVID-19 in HCWs after adjusting for age and sex (Table 2). When never-smoker respondents were accepted as the reference, smoking was found to be inversely related to COVID-19 in HCWs (OR 0.38, 95\%CI 0.23-0.63). The types of PPE significantly associated with lower COVID-19 infection in HCWs were wearing aprons and goggles after the adjustment.

\section{DISCUSSION}

The number of HCWs contracting COVID-19 has increased globally during the pandemic, in line with the total number of infected people. In Figure 1, the peak in weekly diagnoses of HCWs corresponded to that of new COVID-19 diagnoses in Turkey during November and December 2020. Lan et al. ${ }^{8}$ also found a relationship between COVID-19 infection rates in HCWs and the infection rates in their residential community. Wu et al. ${ }^{9}$ evaluated HCW and general population infection data in Ireland and demonstrated a close relationship. The findings of this study, similar to those in the wider literature, point to the importance of community-level measures together with workplace measures to protect HCWs.

According to the findings, occupational characteristics, including working at the onset of the outbreak, not working at the time of the survey, and COVID-19 history in colleagues, 


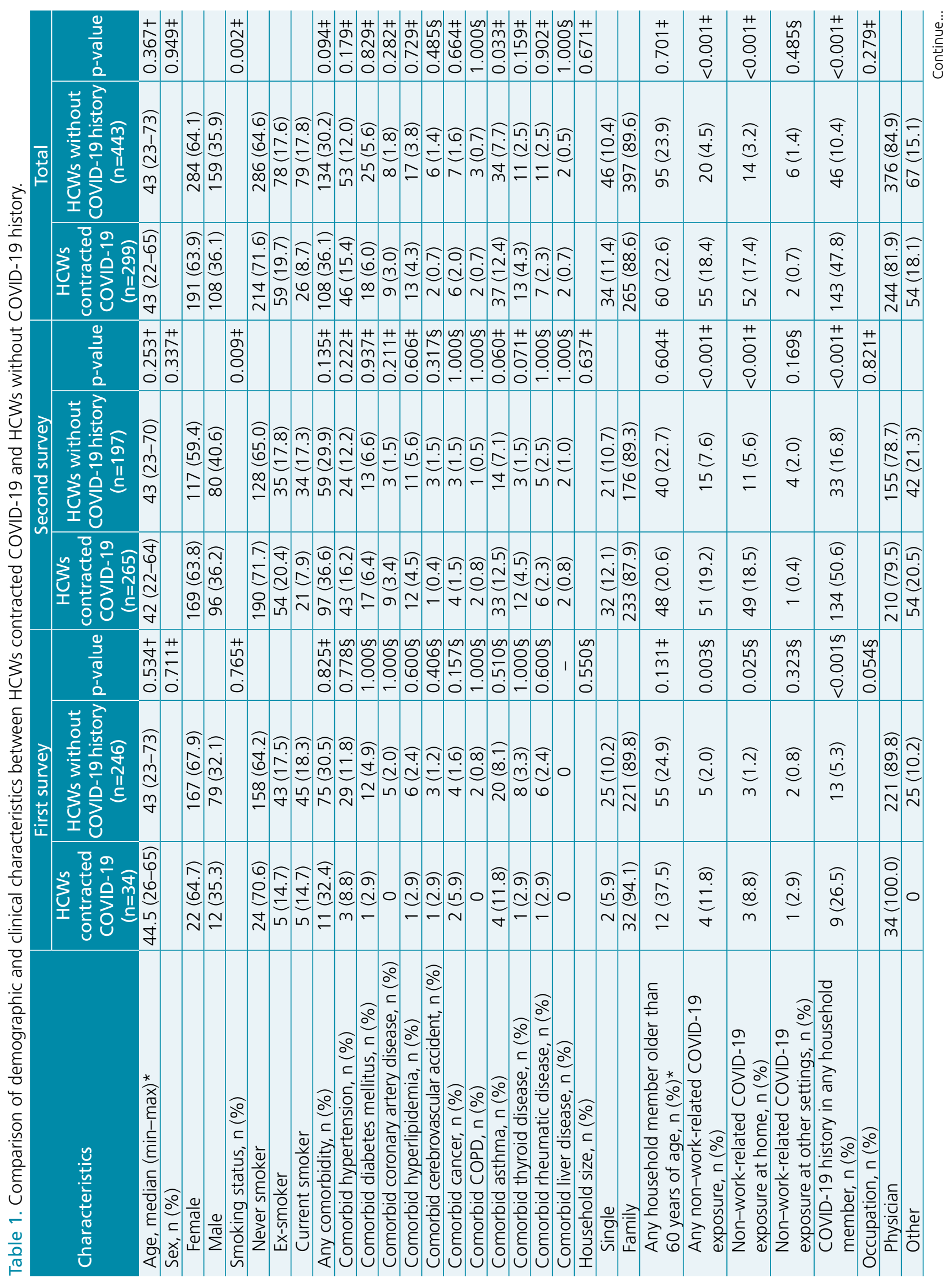




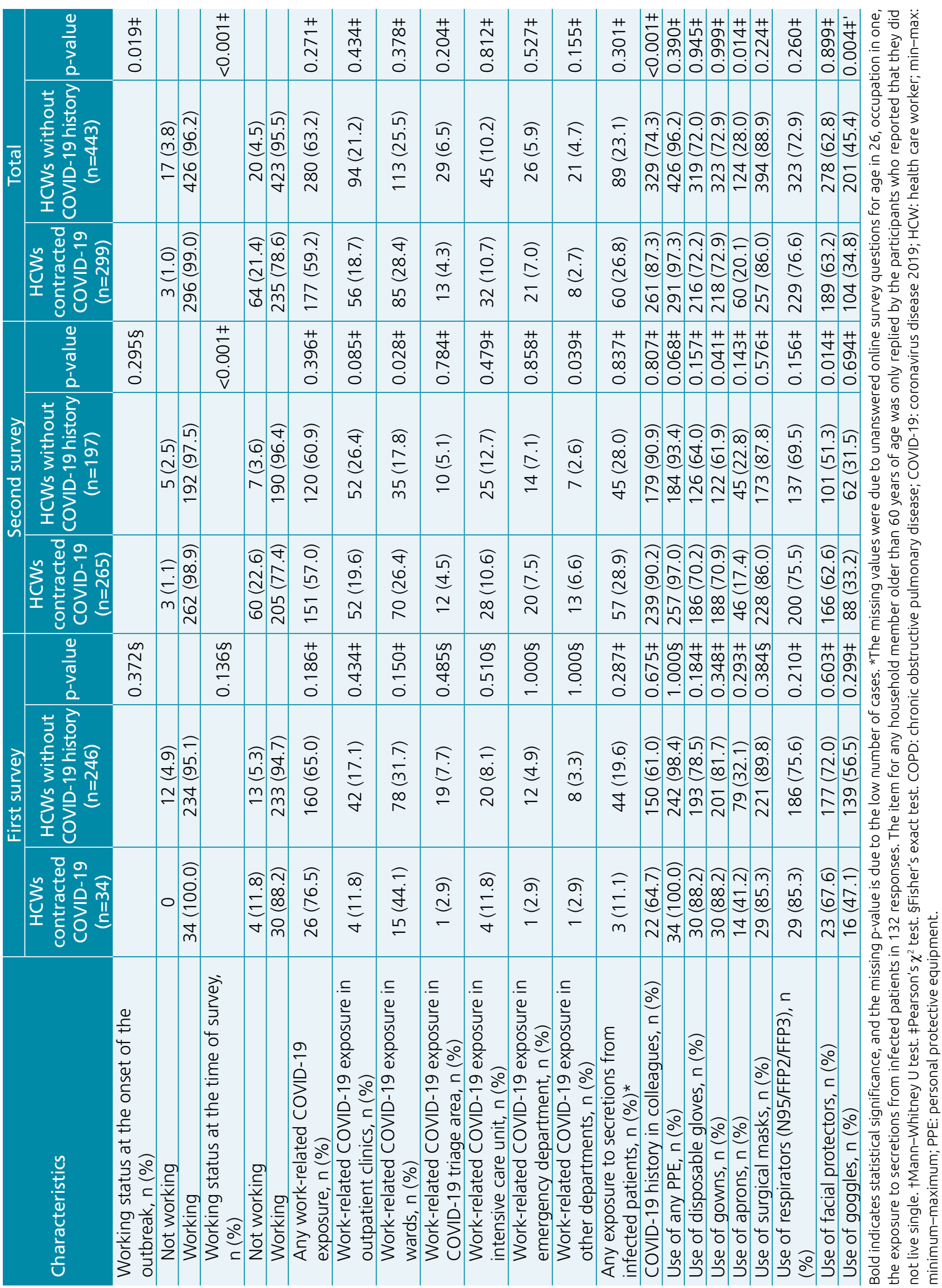


Table 2. Crude and age- and sex-adjusted logistic regression analysis of the association between selected parameters and COVID-19 in health care workers.

\begin{tabular}{|c|c|c|c|c|c|}
\hline & \multicolumn{3}{|c|}{ Crude analysis } & \multicolumn{2}{|c|}{ Adjusted analysis } \\
\hline & $\mathrm{n}$ & OR $(95 \% \mathrm{Cl})$ & $p$-value & OR $(95 \% \mathrm{Cl})$ & $\mathrm{p}$-value \\
\hline Smoking status & 716 & & & & \\
\hline Never smoker & & 1.00 (ref) & & 1.00 (ref) & \\
\hline Ex-smoker & & $0.99(0.67-1.45)$ & 0.947 & $1.04(0.69-1.55)$ & 0.868 \\
\hline Current smoker & & $0.38(0.23-0.63)$ & $<0.001$ & $0.38(0.23-0.63)$ & $<0.001$ \\
\hline Asthma & 716 & $1.62(0.98-2.69)$ & 0.060 & $1.66(1.00-2.75)$ & 0.050 \\
\hline Working at the onset of the outbreak & 716 & $3.88(1.13-13.37)$ & 0.032 & $3.76(1.09-12.98)$ & 0.036 \\
\hline Any work-related COVID-19 exposure & 716 & $0.84(0.62-1.15)$ & 0.276 & $0.84(0.62-1.14)$ & 0.258 \\
\hline Any exposure to secretions from infected patients & 588 & $1.14(0.77-1.68)$ & 0.522 & $1.11(0.75-1.65)$ & 0.607 \\
\hline Not working at the time of survey & 716 & $5.67(3.34-9.64)$ & $<0.001$ & $5.69(3.35-9.67)$ & $<0.001$ \\
\hline COVID-19 history in colleagues & 716 & $2.29(1.52-3.44)$ & $<0.001$ & $2.27(1.51-3.41)$ & $<0.001$ \\
\hline Non-work-related COVID-19 exposure & 716 & $4.66(2.72-7.99)$ & $<0.001$ & $4.72(2.74-8.14)$ & $<0.001$ \\
\hline COVID-19 exposure at home & 716 & $6.45(3.50-11.90)$ & $<0.001$ & $6.52(3.52-12.08)$ & $<0.001$ \\
\hline COVID-19 history in any household member & 716 & $7.98(5.42-11.74)$ & $<0.001$ & $8.16(5.52-12.08)$ & $<0.001$ \\
\hline Use of disposable gloves & 716 & $1.03(0.74-1.44)$ & 0.853 & $1.00(0.71-1.40)$ & 0.976 \\
\hline Use of gowns & 716 & $0.96(0.69-1.35)$ & 0.823 & $0.96(0.68-1.34)$ & 0.796 \\
\hline Use of aprons & 716 & $0.64(0.45-0.92)$ & 0.017 & $0.62(0.43-0.90)$ & 0.011 \\
\hline Use of surgical masks & 716 & $0.80(0.51-1.26)$ & 0.340 & $0.80(0.51-1.27)$ & 0.341 \\
\hline Use of respirators (N95/FFP2/FFP3) & 716 & $1.19(0.84-1.68)$ & 0.323 & $1.17(0.82-1.65)$ & 0.388 \\
\hline Use of facial protectors & 716 & $1.07(0.78-1.46)$ & 0.676 & $1.06(0.78-1.44)$ & 0.725 \\
\hline Use of goggles & 716 & $0.64(0.47-0.88)$ & 0.005 & $0.64(0.47-0.87)$ & 0.005 \\
\hline
\end{tabular}

Cl: confidence interval; COVID-19: coronavirus disease 2019; HCW: health care worker; OR: odds ratio; ref: reference. Bold indicates statistical significance.

align with the COVID-19 in HCWs. The study also observed an inverse relationship between the use of aprons and goggles and COVID-19 in HCWs. Even in the early days of the pandemic, occupational risk factors in HCWs regarding COVID19 were documented. According to a rapid review, the hospital division where the HCW worked and the use of PPE, particularly masks, were the parameters found related to COVID-19 in $\mathrm{HCW}^{10}$. In an evaluation of 4,664 Swiss HCWs, Kahlert et al. ${ }^{11}$ showed that close contact with patients with COVID-19 and exposure to co-workers with COVID-19 were related to COVID-19 in HCWs. This study also evaluated the types of PPE used during close contact and revealed an inverse relationship between the use of any face mask, gloves, gown, and goggles and COVID-19, but a direct relationship with nonusage of PPE. Our results are compatible with the similar studies in the literature.

We observed that the variables associated with COVID-19 in HCWs, other than occupational characteristics, included non-work-related COVID-19 exposure, COVID-19 exposure at home, and a COVID-19 history among household members. Several studies also evaluated nonoccupational factors in HCWs. Kahlert et al. ${ }^{11}$ revealed that a COVID-19-positive household member and a history of visiting a COVID-19 hotspot were related to COVID-19 in HCWs. Çelebi et al. ${ }^{12}$ demonstrated that having a SARS-CoV-2-positive household member bore a significant relationship to COVID-19 in HCWs. Combining these results, we consider that nonoccupational risk factors are also integral for COVID-19 in HCWs depending on the increased community transmission during the outbreak.

The results showed a statistically significant difference in smoking status between HCWs who contracted COVID-19 and those without a COVID-19 history. Moreover, current smoking is inversely related to COVID-19 in HCWs (OR=0.38, 95\% CI: $0.23-0.63$ ) when nonsmoking respondents were accepted as the reference. Since the beginning of the pandemic, the association between smoking status and COVID-19 has been investigated, and alternative biological mechanisms have been proposed to suggest an increased or decreased risk for COVID-19 due to 
smoking ${ }^{13}$. The number of studies and meta-analyses documenting the relationship between smoking and the severity of COVID-19 has increased ${ }^{14}$. However, Kahlert et al. ${ }^{11}$ showed a similar result to this study for active smoking. More substantial prospective studies are required to document if the risk of contracting COVID-19 changes according to the smoking status and relevant mechanisms.

According to the results, $59.2 \%$ of $\mathrm{HCW}$ who contracted COVID-19 were asymptomatic. A meta-analysis estimated that $40 \%$ of RT-PCR positive HCWs were asymptomatic ${ }^{15}$. The results also showed that the most prevalent symptoms were cough, headache, and loss of smell and/or taste. Similarly, an observational study found the prevalence of cough as $82.2 \%$ in 185 symptomatic and COVID-19-positive Belgian HCW $\mathrm{s}^{16}$. Despite varying frequencies according to the study design, these results indicate the need for a screening program for both symptomatic and asymptomatic HCWs regarding the risk status.

The strengths of this study include representation of the national profile due to a wide range of participants from different provinces of Turkey, more varied items investigating both occupational and nonoccupational parameters in HCWs in terms of contracting COVID-19, and a two-phase design to evaluate temporal change over time. However, the study has some limitations. Online surveys have classical constraints about the percentage of participation, the representativeness of the sample of the wider population, and data collection and quality, despite a relatively longer duration for the data collection being applied in both phases. The nature of the data collection method may favor the participation of HCWs with a history of the nonsevere disease, although the survey questions did not address the severity of COVID-19 in HCWs. The cumulative probability of exposing occupational and nonoccupational risks during the pandemic increases; however, most survey questions for occupational and nonoccupational parameters did not include a temporal and quantitative evaluation. This strategy might have caused a limitation in the grading of the risks.

\section{CONCLUSIONS}

Occupational and nonoccupational parameters are related to COVID-19 in HCWs. Active surveillance, including the diagnosis of both symptomatic and asymptomatic HCWs, and documenting and controlling occupational and nonoccupational risks should be maintained. Future prospective studies may document the changes related to dynamic features of an ongoing pandemic.

\section{ACKNOWLEDGMENTS}

The authors thank the members of the Executive Committee of the Turkish Thoracic Society for their collaboration and support. The preliminary findings of this study on the data of the first survey were presented as an oral presentation at the Turkish Thoracic Society's 23rd Annual Congress (Virtual Congress, October 15-18, 2020).

\section{AUTHORS" CONTRIBUTIONS}

AS: Conceptualization, Data curation, Formal Analysis, Investigation, Methodology, Project administration, Supervision, Visualization, Writing - original draft. ZNT: Data curation, Formal Analysis, Methodology, Writing - original draft. CS: Conceptualization, Data curation, Methodology, Supervision, Writing - review \& editing. PMA: Conceptualization, Formal Analysis, Investigation, Methodology, Project administration, Supervision, Writing - review \& editing.

\section{REFERENCES}

1. Cespedes MDS, Souza JCRP. Sars-CoV-2: a clinical update - II. Rev Assoc Med Bras (1992). 2020;66(4):547-57. https://doi. org/10.1590/1806-9282.66.4.547

2. Koh D. Occupational risks for COVID-19 infection. Occup Med (Lond). 2020;70(1):3-5. https://doi.org/10.1093/occmed/ kqaa036

3. Fellows of the Collegium Ramazzini. 24th Collegium Ramazzini Statement: prevention of work-related infection in the COVID-19 pandemic. Ann Glob Health. 2020;86(1):79. https:// doi.org/10.5334/aogh.2929

4. Keskinkiliç B, Shaikh I, Tekin A, Ursu P, Mardinoglu $A$, Mese EA. A resilient health system in response to Coronavirus disease 2019: experiences of Turkey. Front Public Health. 2021;8:577021. https://doi.org/10.3389/ fpubh.2020.577021
5. Vatan A, Güçlü E, Öğütlü A, Kibar FA, Karabay O. Knowledge and attitudes towards COVID-19 among emergency medical service workers. Rev Assoc Med Bras (1992). 2020;66(11):1553-9. https://doi.org/10.1590/1806-9282.66.11.1553

6. Cakir B. COVID-19 in Turkey: lessons learned. J Epidemiol Glob Health. 2020;10(2):115-7. https://doi.org/10.2991/ jegh.k.200520.001

7. World Health Organization. WHO Coronavirus (COVID-19) Dashboard [internet]. Geneva: World Health Organization; 2021. [cited on Apr. 4, 2021]. Available from: https://covid19.who.int.

8. Lan FY, Filler R, Mathew S, Buley J, lliaki E, Bruno-Murtha LA, et al. Sociodemographic risk factors for COVID-19 infection among Massachusetts healthcare workers: a retrospective cohort study. Infect Control Hosp Epidemiol. 2021:1-6. https:// doi.org/10.1017/ice.2021.17 
9. Wu D, Mac Aonghusa P, O'Shea DF. Correlation of national and healthcare workers COVID-19 infection data; implications for large-scale viral testing programs. PLoS One. 2021;16(4):e0250699. https://doi.org/10.1371/journal. pone.0250699

10. Chou R, Dana T, Buckley DI, Selph S, Fu R, Totten AM. Epidemiology of and Risk Factors for Coronavirus Infection in Health Care Workers: A Living Rapid Review. Ann Intern Med. 2020;173(2):120-36. https://doi.org/10.7326/M20-1632

11. Kahlert CR, Persi $R$, Güsewell $S$, Egger T, Leal-Neto OB, Sumer J, et al. Non-occupational and occupational factors associated with specific SARS-CoV-2 antibodies among hospital workers - A multicentre cross-sectional study. Clin Microbiol Infect. 2021;27(9):1336-44. https://doi.org/10.1016/j.cmi.2021.05.014

12. Çelebi G, Pişkin N, Bekleviç AÇ, Altunay $Y$, Keleş AS, Tüz MA, et al. Specific risk factors for SARS-CoV-2 transmission among health care workers in a university hospital. Am J Infect Control. 2020;48(10):1225-30. https://doi.org/10.1016/j.ajic.2020.07.039
13. Polverino F. Cigarette smoking and COVID-19: a complex interaction. Am J Respir Crit Care Med. 2020;202(3):471-2. https://doi.org/10.1164/rccm.202005-1646LE

14. Haddad C, Malhab SB, Sacre H, Salameh P. Smoking and COVID-19: a scoping review. Tob Use Insights. 2021;14:1179173X21994612. https://doi.org/10.1177/1179173X21994612

15. Gómez-Ochoa SA, Franco OH, Rojas LZ, Raguindin PF, Roa-Díaz ZM, Wyssmann BM, et al. COVID-19 in healthcare workers: a living systematic review and meta-analysis of prevalence, risk factors, clinical characteristics, and outcomes. Am J Epidemiol. 2020;190(1):161-75. https:// doi.org/10.1093/aje/kwaa191

16. Van Loon N, Verbrugghe $M$, Cartuyvels R, Ramaekers D. Diagnosis of COVID-19 based on symptomatic analysis of hospital healthcare workers in belgium: observational study in a large Belgian Tertiary Care Center during early COVID-19 outbreak. J Occup Environ Med. 2021;63(1):27-31. https:// doi.org/10.1097/JOM.0000000000002015 